\title{
An Update on the Role of Nrf2 and its Activators in Diseases Associated with Oxidative Stress
}

\author{
DEEPA SUGUMAR, J. SARAVANAN *, R. EMDORMI AND T.K. PRAVEEN \\ Department of Pharmacology, JSS College of Pharmacy, Ooty, Tamil Nadu-643001, India
}

Sugumar et al.: Nrf2 Diseases-related oxidative stress

\begin{abstract}
A wide range of experimental and observational studies have established the irrefutable role of oxidative stress in the pathogenesis of a plethora of diseases either directly or indirectly. Several factors ranging from radiation, pollution, high fat and sugar diet, alcohol consumption, smoking, tobacco consumption and even certain drugs contribute to oxidative stress. The imbalance between oxidant and antioxidant levels remain the underlying cause of oxidative stress. When the levels of oxidants outweigh the levels of antioxidants it leads to formation of free radicals like hydroxyl, superoxide, alkoxyl, peroxyl, glutathiyl, tocopheroxyl, hydroperoxyl and ascorbate. These free radical species play a definitive role in the pathogenesis of neurological diseases, malignancies, cardiovascular, respiratory and liver diseases. Nuclear factor erythroid-2-related factor 2 is a master transcription factor belonging to the leucine zipper family. Kelch-like ECH-associated protein 1 is a repressor of nuclear factor erythroid-2-related factor 2 under basal or normal conditions, which is responsible for cytoplasmic sequestration and proteosomal degradation of nuclear factor erythroid-2-related factor 2 via ubiquitination. The activation of nuclear factor erythroid-2-related factor 2/Kelch-like ECH-associated protein 1/antioxidant response element signalling pathway regulates the expression of numbers of genes that are cytoprotective, antioxidative and detoxificative in action. This article reviews the potential therapeutic role of nuclear factor erythroid-2related factor 2 activators in prevention and treatment of those diseases in which oxidative stress plays a definitive role in the pathogenesis.
\end{abstract}

Key words: Nuclear factor erythroid-2-related factor 2, keap1, antioxidant response element, oxidative stress, free radicals

Oxidativestress(OS)playsamajorrolein the development and progression of a wide range of diseases including Alzheimer disease, cancer, atherosclerosis ${ }^{[1]}$, chronic kidney disease ${ }^{[2]}$, multiple sclerosis, Niemann-Pick C, Parkinson's disease, Friedreich's ataxia, Huntington's disease (HD), infantile neuroaxonal degeneration, neurodegeneration with brain iron accumulation (NBIA) and lipid metabolism deregulation syndrome like Zellweger syndrome ${ }^{[3]}$. An assumption also exists currently that OS could cause cancer in patients with Type 2 Diabetes Mellitus (T2DM $)^{[4,5]}$. The neurons and oligodendrocytes of the central nervous system are particularly more susceptible to OS ${ }^{[6]}$. Reactive Oxygen Species (ROS) include free radicals such as superoxide $\left({ }^{\circ} \mathrm{O}_{2}^{-}\right)$, peroxyl $\left({ }^{\circ} \mathrm{RO}_{2}\right)$, hydroxyl $\left({ }^{\circ} \mathrm{OH}\right)$, hydroperoxyl $\left(\mathrm{HRO}_{2}\right)$, and nonradical species such as hydrochlorous acid $(\mathrm{HOCl})$ and hydrogen peroxide $\left(\mathrm{H}_{2} \mathrm{O}_{2}\right)^{[7]}$. ROS are the small oxygen-containing end-products of aerobic metabolism, which are highly reactive ${ }^{[8]}$. The

*Address for correspondence

E-mail: getsarwan@gmail.com

March-April 2020 increase in the level of these species often disrupt the normal oxidative-redox system ${ }^{[9]}$. In the recent times several works have reported the relationship between the nuclear factor E2-related factor 2 (Nrf2) and $\mathrm{OS}^{[10-13]}$. Nrf2, a nuclear transcriptional factor is observed to show a protective role in cell defence and survival against xenobiotics and $\mathrm{OS}^{[14]}$ via the control and regulation of the production of number of genes and enzymes that function as antioxidants. Some of the antioxidant enzymes produced through the activation of Nrf2 are, superoxide dismutase (SOD), sulfiredoxin (Srx), peroxiredoxin (Prx), GSH peroxidase (GPx) and sestrin2 (Sesn2), which are involved in catabolism of

This is an open access article distributed under the terms of the Creative Commons Attribution-NonCommercial-ShareAlike 3.0 License, which allows others to remix, tweak, and build upon the work non-commercially, as long as the author is credited and the new creations are licensed under the identical terms

Accepted 05 Januaryy 2020

Revised 13 February 2020

Received 27 December 2019 Indian J Pharm Sci 2020;82(2):184-192 

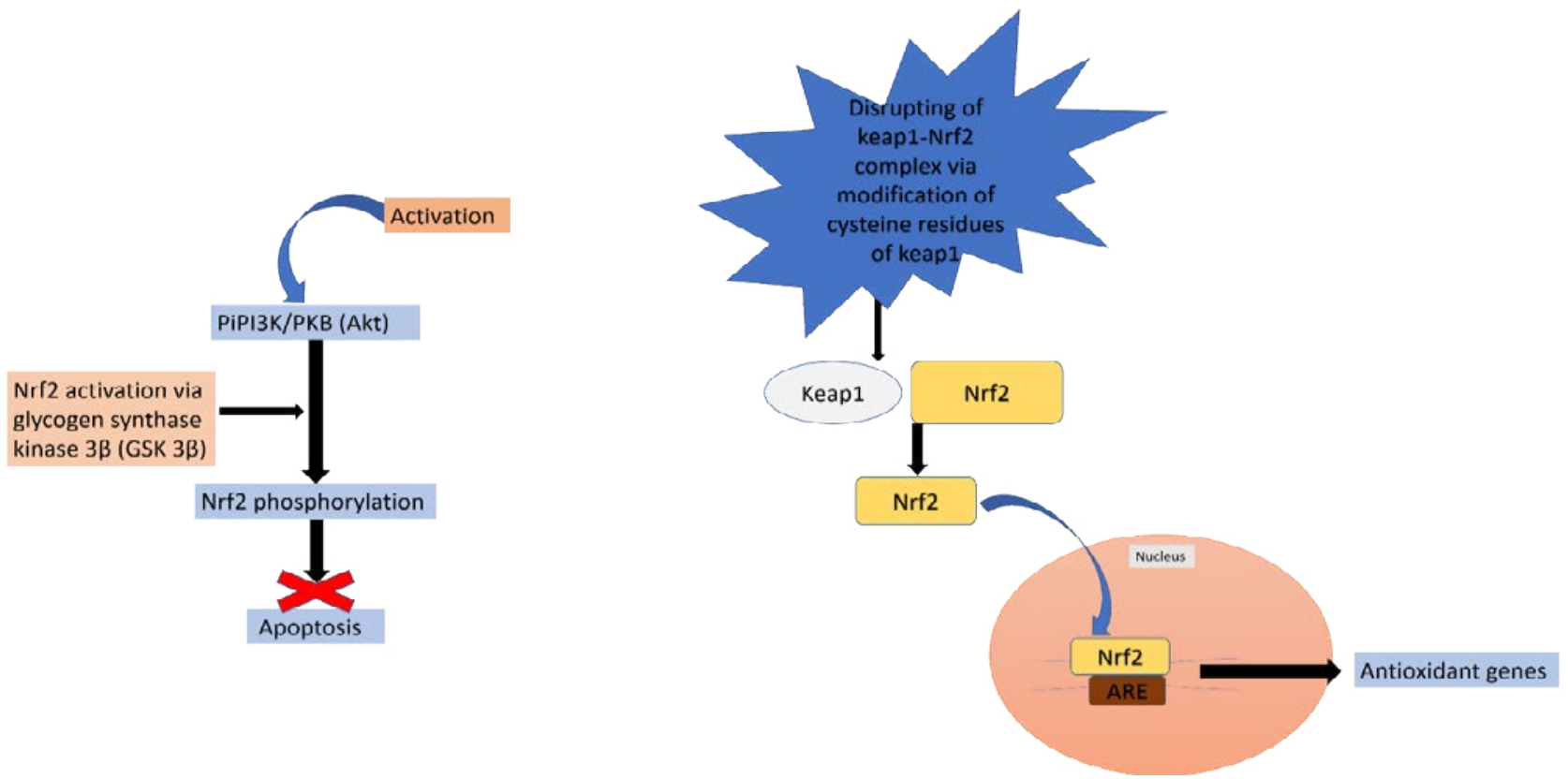

Fig. 1: Mechanism of Nrf2 activators

free radicals into hydrogen peroxide, which is further broken down into water and oxygen by the enzyme catalase. Nrf2 also regulates the synthesis of GSH with glutamate-cysteine ligase modifier (GCLM) subunit and glutamate-cysteine ligase catalytic subunit (GCLC). Nrf2 also increases the redox cysteine/ glutamate transport and peroxidase, which is a hemoprotein that uses hydrogen peroxide to oxidize a number of substrates. Nrf2 also regulates the levels of catalase, a major defence enzyme against OS which is responsible for conversion of hydrogen peroxide into water and oxygen, and NADPH production by glucose-6-phosphate dehydrogenase (G6PD) and 6-phosphogluconate dehydrogenase (6PGD). Nrf2 also augments the activity of stress response proteins, such as heme oxygenase-1 (HO-1), which affects a variety of cellular functions and upregulation of metal chelators, metallothionein1/2 (MT1/2) and ferritin. Non-enzymatic antioxidants like $\mathrm{NAD}(\mathrm{P}) \mathrm{H}$ quinone oxoreductase (NQO1), GPx, glutathione S-transferase, and thiols are also upregulated by $\mathrm{Nrf}^{[2,15,16]}$. It is clear that Nrf2 plays a role in protecting cells against OS and this property of Nrf2 makes it an interesting target in several diseases where OS plays a significant role in pathogenesis.

\section{Overview of NRF2- Kelch-like ECH-associated protein 1 (Keap1):}

$\mathrm{Nrf} 2$ is a master transcription factor belonging to the leucine zipper family ${ }^{[17]}$. It contains a basic leucine-zipper (bZip) domain at the C-terminus and participates in the formation of heterodimers with other
bZip proteins like muscle aponeurosis fibromatosis $(\mathrm{MAF})^{[18]}$. Under normal conditions Keap1, a cytoplasmic protein associated with the Cullin3 (Cul3) based E3 ligase complex, binds to Nrf2 leading to proteosomal degradation of $\mathrm{Nrf2}$ via ubquitination ${ }^{[19,20]}$. Nrf2 consists of 6 highly conserved homology domains named Neh1 to Neh6 (Nrf2-ECH homology). Among all, in fibroblasts, Neh2 is a dependent transactivation domain and Neh4 and Neh5 are 2 independent activation domains ${ }^{[21]}$. Keap1 and $\beta$-transducin repeatscontaining proteins $(\beta-\mathrm{TrCP})$ are $\mathrm{E} 3$ ubiquitin ligase adaptor proteins that regulates $\mathrm{Nrf} 2$ intracellularly by association with its two degradation domains, Neh2 and Neh6 ${ }^{[22]}$. Cytoplasmic localization of Nrf2 is determined by Neh2, recruitment of transcription factors and other canonical protein needed for gene expression are determined by Neh4 and Neh5. Kelch domain and the bric-a-brac, tram track, broad-complex (BTB) domain are the two domains of Keap1. Kelch binds to actin thereby tie the Keap1-Nrf2 complex to the cytoskeleton and BTB is important for Keap1-protein dimerization $^{[23]}$. The conformational modifications by ectopic and endogenous electrophiles (residues C151, C273 and C288) from the sulfhydryl groups of Keap1 allow Nrf2 to escape Keap1-dependent degradation. Thus, Nrf2 accumulates in the nucleus and activates ARE-genes. On the other hand, $\beta$-TrCP, which is a homodimeric E3 ligase adapter also signals Nrf2 modification via phosphorylation by glycogen synthase kinase-3 (GSK-3) that promotes proteasomal degradation. Therefore, GSK-3/ $\beta$-TrCP also serves as a potential target to increase the activity of $\mathrm{Nrf}^{224,25]}$ 


\section{Mechanism of action of nrf 2 activators:}

Several mechanisms have been proposed for activation of Nrf2 at the molecular level. One hypothesis is that activation of $\mathrm{PI} 3 \mathrm{~K} / \mathrm{PKB}$ (Akt) signalling leads to phosphorylation of Nrf2 by glycogen synthase kinase $3 \beta$. Akt, a downstream target of PI3K prevents cells form undergoing apoptosis. Another hypothesis is that the degradation of Nrf2 is regulated by a protein called Keap1. The binding of keap1 to Nrf2 leads to proteasomal degradation of Nrf2. Disruption of this complex via modification of cysteine residues within the Keap1 destabilizes the Nrf2-Keap1 complex thereby allowing the free translocation and nuclear accumulation of Nrf2. Nrf2 once inside the nucleus binds to ARE and activates the transcription of cytoprotective genes ${ }^{[26-29]}$.

\section{Diseases-related OS:}

An overproduction of ROS and the deficiency in the levels of antioxidants leads to OS (fig. 2) ${ }^{[30]}$. In alcohol abuse, there is a surge in OS markers and increase in lipid peroxidation products (F2-isoprostane and 4-hydroxy-2-nonenal) but depletion of antioxidants like glutathione (GSH) and vitamins $\mathrm{E}$ and $\mathrm{C}$ (fig. 3). In hepatitis, hepatocellular and cholestatic dysfunction, DNA damage, lipid damage and mitochondrial dysfunction occurs as a result of OS (fig. 4). In renal disease like $\mathrm{CKD}$, end-stage renal disease and chronic inflammation, there is an impairment of activation of Nrf2 and diminished antioxidant defence (fig 4) ${ }^{[31]}$. In T2DM the Nrf2 induction suppressed the formation of DNA adducts via ROS-induction, intracellular ROS formation, apoptosis of pancreatic $\beta$-cells within the islets as well as a reduction in inflammation via NF $\mathrm{B}$ pathways. Fig. 5 denotes the role of ROS and the effects of Nrf2 activation in regulation of OS in $\mathrm{T}_{2} \mathrm{DM}^{[32]}$. ROS has also been reported to increase apoptosis in Sertoli cells by decreasing the expression of blood-testis barrier (occludin, N-cadherin, zonula occludens- 1 and $\beta$-catenin) and disordering the F-actin spatial arrangement. This is believed to be because of the upregulation of Jun N-terminal (JNK), extracellular signal regulatory kinase (ERK), p38 mitogen-activated protein kinase (MAPK) and downregulation of Nrf2. This, indicates that ROS-MAPK-Nrf2 is involved in spermatogenesis dysfunction ${ }^{[33]}$. A similar study on prepubertal testicular injury has suggested the role of OS increase through inhibition of Nrf2-mediated antioxidant signalling pathway ${ }^{[34]}$.

In $\mathrm{AD}, \mathrm{OS}$ and cytotoxicity via the alteration of mitochondrial membrane potential by amyloid beta $(A \beta)$, and the complex of $A \beta 42$ and copper (I) ion leads to reduced oxygen level and generation of $\mathrm{H}_{2} \mathrm{O}_{2}$. Interestingly, the study of AD using Drosophila model showed that there was a decrease in the level of A $\beta 42$ peptide levels upon down regulation of Keap1, suggesting that reducing the activity of Keap1 may clear $A \beta 42$ peptide via protein degradation. In PD, aggregation of $\alpha$-synuclein protein, loss of function of parkin protein and mutation of DJ-1 are known to play a major role in the generation of $\mathrm{OS}^{[35,36]}$. In case of $\mathrm{HD}$, OS results due to the effect of mutant huntingtin $(\mathrm{mHtt})$ that disrupts the Nrf2 signalling pathway ${ }^{[37]}$. Examples of agents that help in combating OS via the activation of $\mathrm{Nrf} 2$ by persulfidation of Keap1 are S-1-propenylmercaptocysteine (CySSPe) and $\mathrm{N}$-acetylcysteine. CySSPe was reported to increase hydrogen sulfide $\left(\mathrm{H}_{2} \mathrm{~S}\right)$ production that persulfidates keap1, enhance the cellular production of GSH and improve GSH:GSSG ratio ${ }^{[38]}$. N-acetylcysteine is

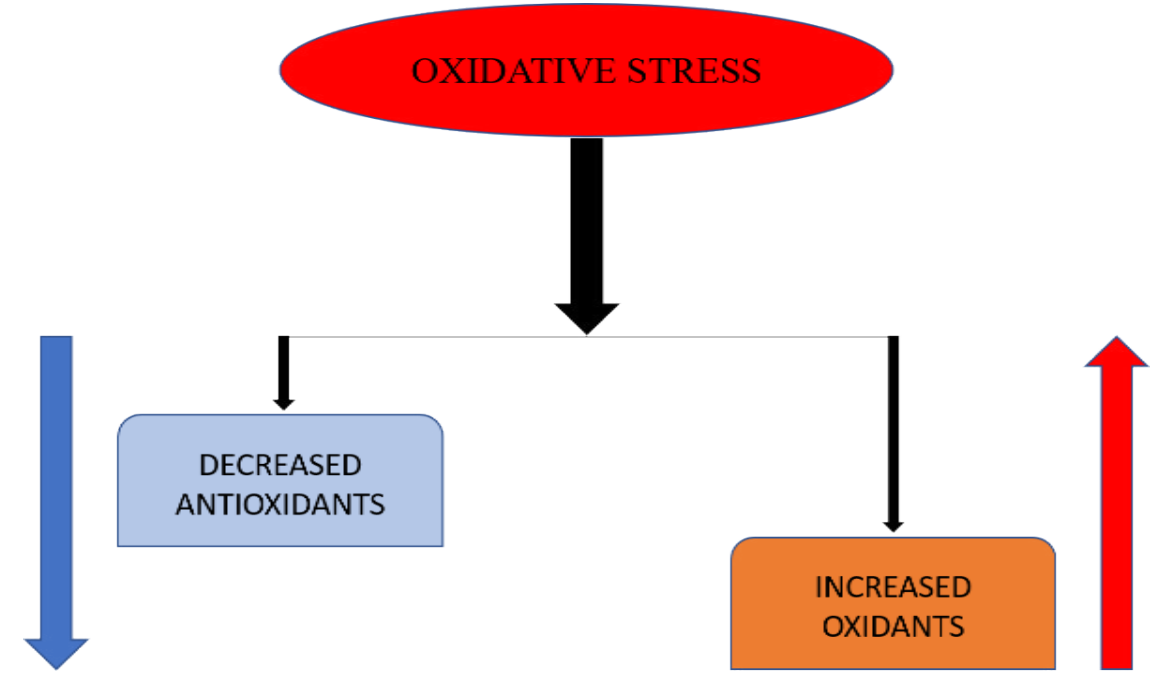

Fig. 2: Oxidative stress due to decreased antioxidants and increased oxidants 


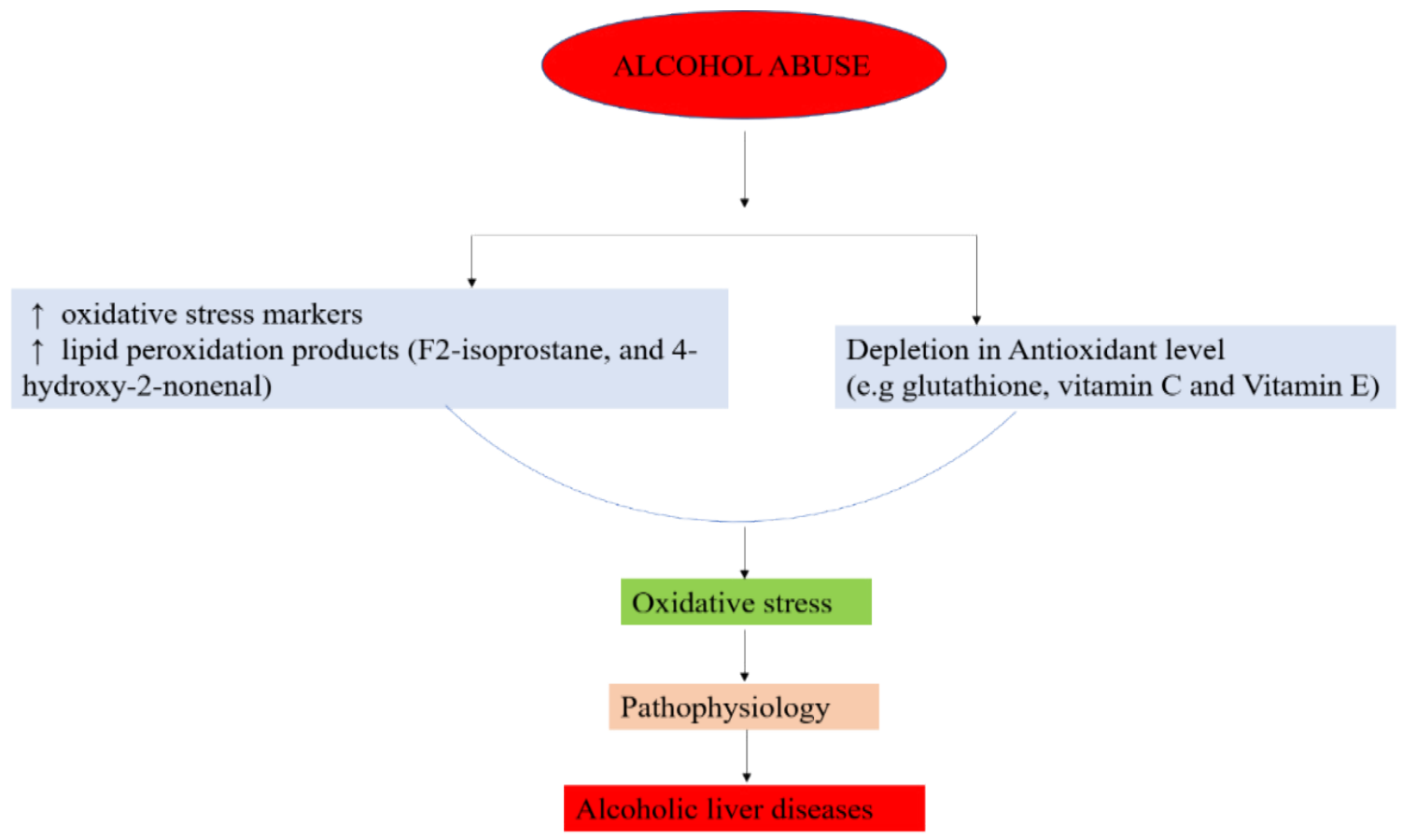

FIG. 3: The increased and decreased in bio markers in alcohol abuse leading to oxidative stress
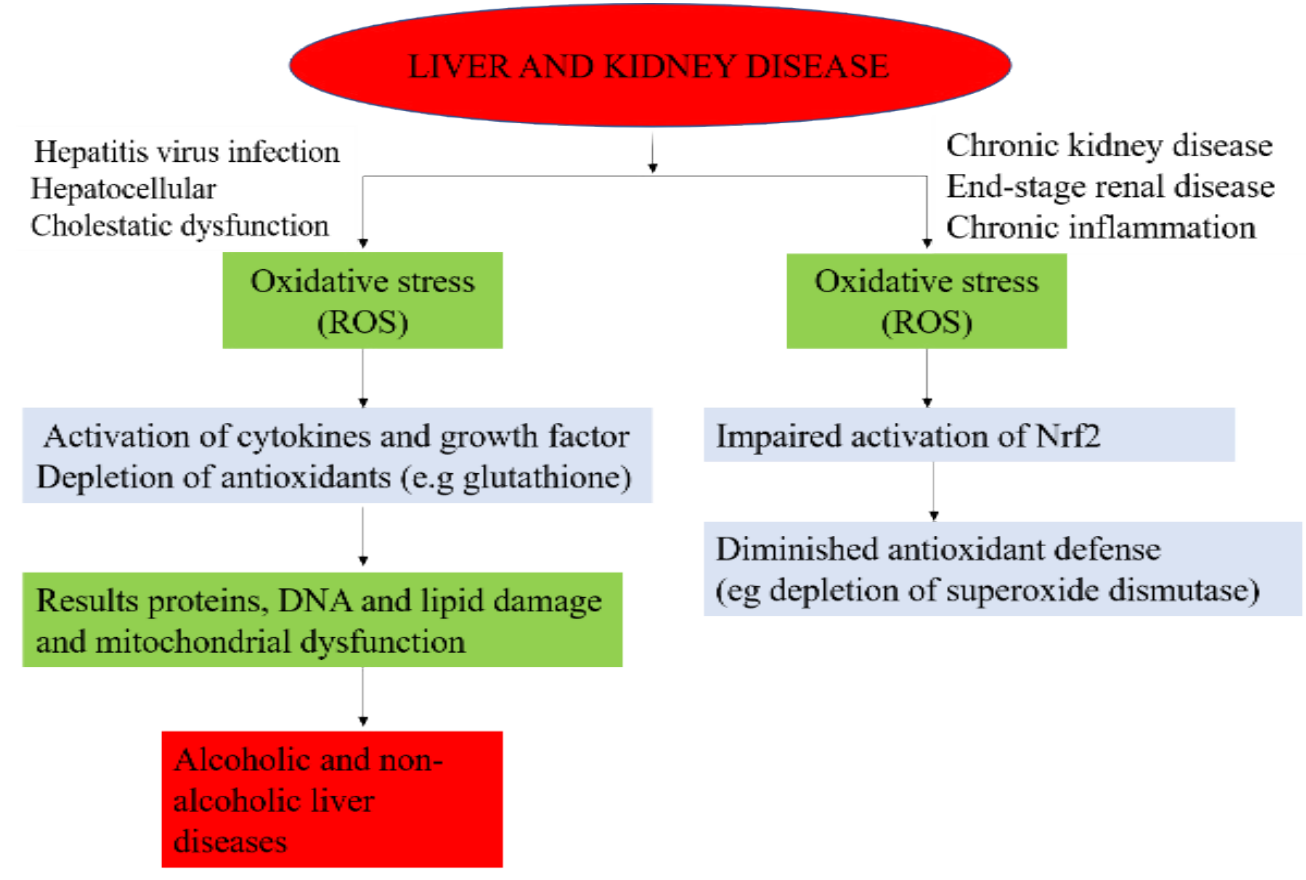

Fig. 4: Impairment of liver and kidney function as a result of oxidative stress

Impairment of liver function in hepatitis, hepatocellular and cholestatic dysfunction and kidney function during CKD and endstage renal disease and chronic inflammation as a result of oxidative stress

a pro-drug that is widely used as an antidote against acetaminophen overdose. It is a cysteine derivative and a direct precursor of GSH with beneficial effects in OSrelated diseases caused by pesticides ${ }^{[39]}$.

\section{NRF2 activators:}

The current consensus is that Nrf2 activators stabilize and promote Nrf2 translocation by inhibiting Keap1 from binding to Nrf2. Nrf2, on translocation into the nucleus leads to production of antioxidant and antiinflammatory substances that decrease OS and inflammation $^{[40]}$. A few examples of $\mathrm{Nrf} 2$ activators are oleanolic acids (OAs), bardoxolone methyl, dimethyl fumarate (DMF) and sulforaphane (SFN), calastrol, ellagic acid, zerumbone, curcumin, quercetin, quercitrin, resveratrol, phenethyl isothiocyante (PEITC), lucidone, forsythoside B and thymoquinone. Fig. 6 represents the chemopreventive/chemoprotective natural products 
and their effects upon Nrf2 activation ${ }^{[41,42]}$. Dimethyl not in astrocytic cells ${ }^{[43,44]}$. The activation of Nrf2 by a fumarate (DMF) has been reported to activate Nrf2 bioactive compound, salvianolic acid B (SalB) has been and increase its activity in neuronal subpopulations but reported to protect dopaminergic neurons in PD via the

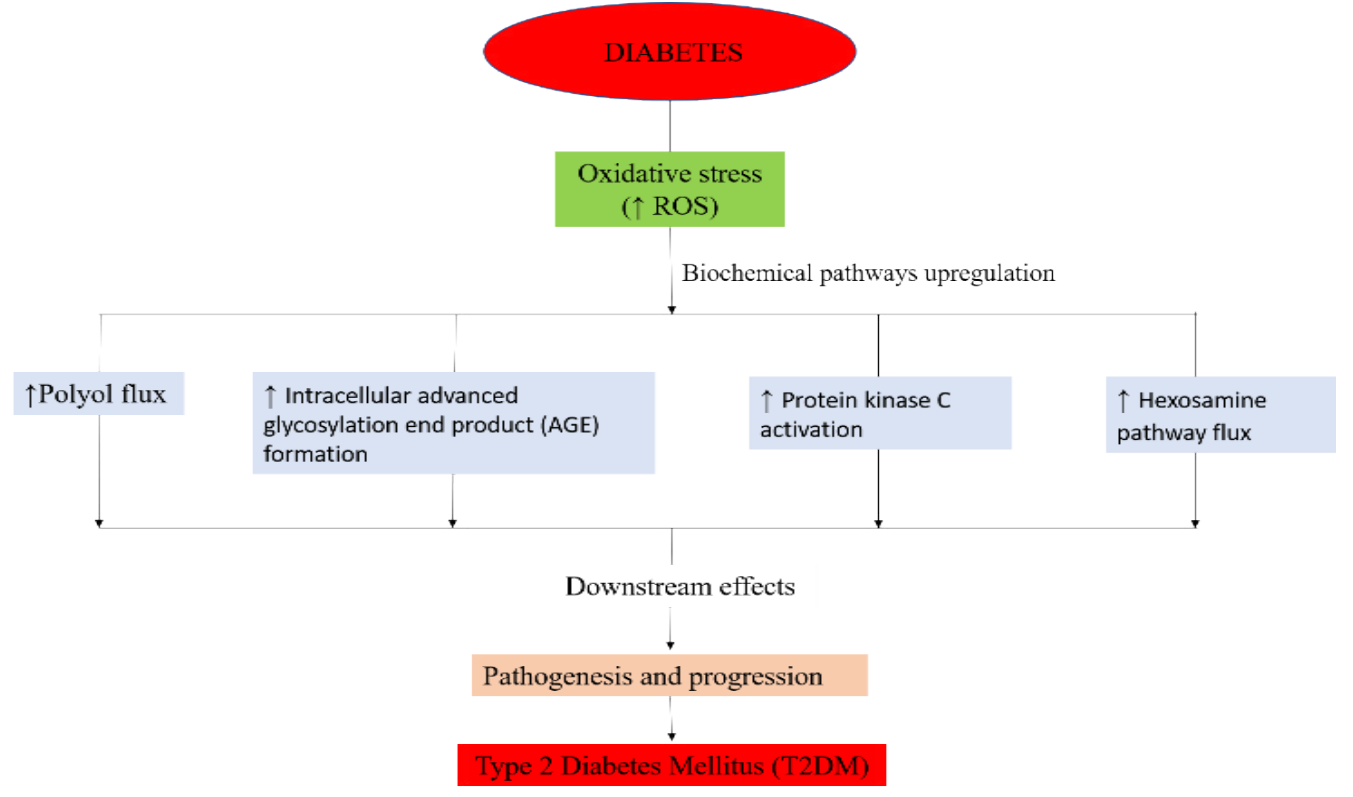

Fig. 5: Role of increased oxidative stress in T2DM

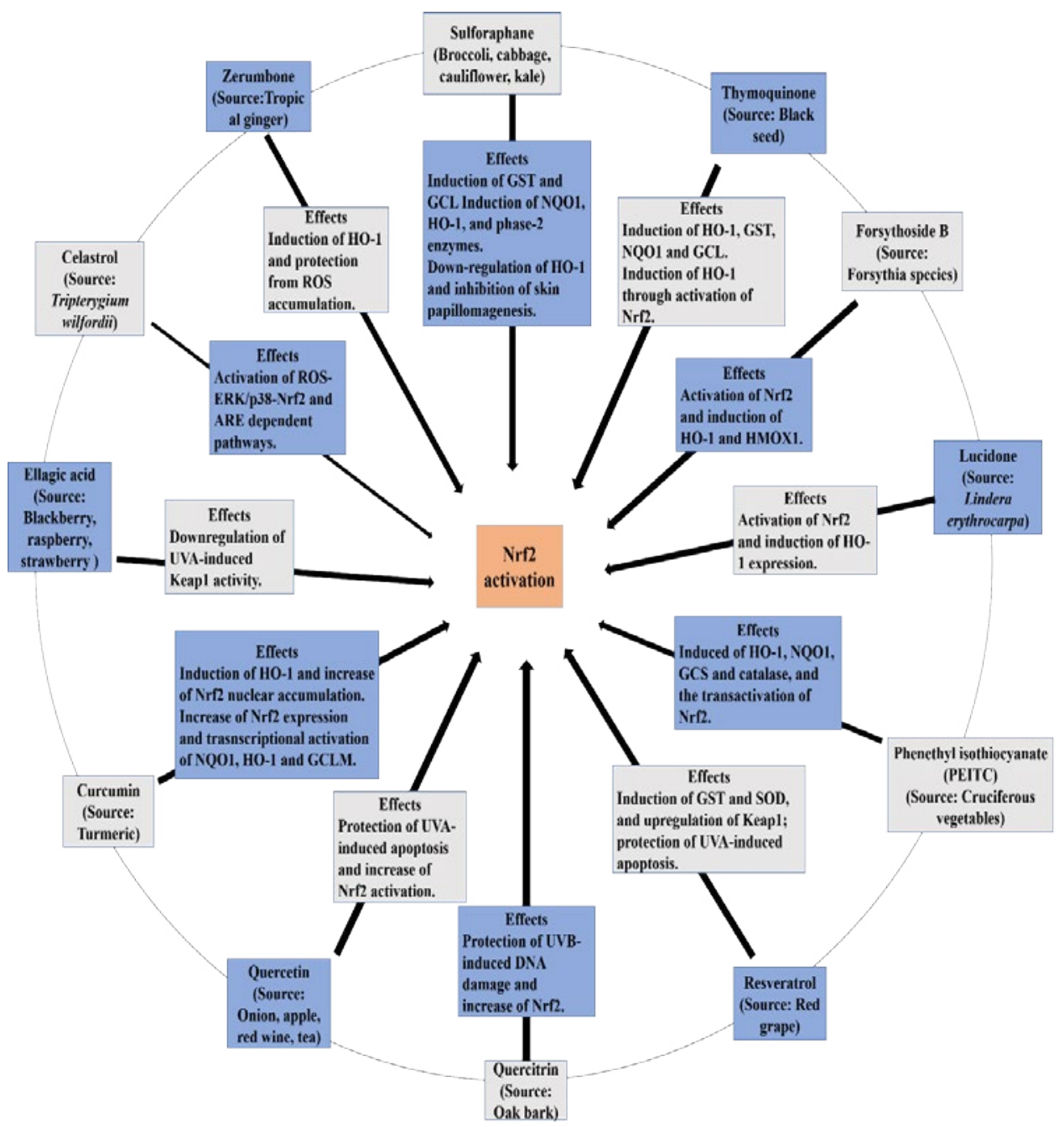

Fig. 6: Chemopreventive/chemoprotective natural products and their effects upon Nrf2 activation 
inactivation of microglia-mediated inflammation and through induction of astrocyte activation-dependent GDNF expression ${ }^{[45]}$. Similarly, in a rat PD model with severe OS induced by 6-hydroxydopamine (6-OHDA) pre-treatment with phloroglucinol, a polyphenol salvaged the decrease in Nrf2 and p-Nrf2 in the nuclear fraction ${ }^{[46]}$. In a similar study in a rat PD model with OHDA as an inducing agent, icariin targets Nrf2 signalling by inhibition of microglia-mediated neuroinflammation ${ }^{[47]}$. Nrf2 plays an vital role in maintaining newly generated hepatocytes during liver repair ${ }^{[48,49]}$. Activation of $\mathrm{Nrf} 2$ by pre-treatment with resveratrol in mice heart has been reported to alleviate the levels of mitochondrial ROS production induced by lipopolysaccharide in cardiomyocytes ${ }^{[50]}$. In another study, pre-incubation of Sertoli cells with luteolin, a natural flavonoid, induced the expression of antioxidant enzymes and increased ARE-luciferase reporter activity via triggering $\mathrm{Nrf} 2$ translocation $^{[51]}$. In case of mice with transverse aortic arch constriction (TAC) model with maladaptive cardiac remodelling and dysfunction, dihydro-CDDO-trifluoroethyl amide (dh404) upregulates myocardial Nrf2 protein expression and inhibit cardiomyocyte hypertrophy and proliferation of cardiac fibroblast in chronic pressure overload, heart failure and maladaptive cardiac remodelling and dysfunction ${ }^{[52]}$. Sulforaphane, the most abundant isothiocyanate has been reported to alleviate or retard the ischemia-reperfusion induced retinal damage and grape seed proanthocyanidin has been shown to have a protective role in diabetic bladder dysfunction via the activation of Nrf2/HO-1 pathway ${ }^{[53,54]}$. Sulforaphane has also been reported to inhibit HIV infection in primary macrophages by reducing the activity of luciferase enzyme and increase the expression of $\mathrm{Nrf}^{[55]}$. In a study conducted by Jazwa et al. the activation of Nrf2 by sulforaphane (SFN) in rats with methyl-4-phenyl1-2-3-6-tetrahydropyridine (MPTP)-induced PD elevates Nrf2 levels in the basal ganglia and a further increase in phase II antioxidant enzymes, HO-1 and $\mathrm{NQO1}^{[56]}$. However, the above results with SFN results were contradicted in clinical studies ${ }^{[41]}$. Synthetic triterpenoid CDDO-MA activates Nrf2 in wild type mouse embryonic fibroblasts by blocking t-butyl hydroperoxide that induce the production of ROS (fig. 7) ${ }^{[57]}$. According to a study in RAW264.7 cells, curcumin upon activation of Nrf2-Keap1 pathway, leads to an increase in activity of antioxidant enzymes and showed a bifunctional activity i.e., lowering the ROS production at a lower dose $(5 \mu \mathrm{M})$ and middle-dose $(10 \mu \mathrm{M})$ whereas it intensified the production of ROS at a high-dose $(20 \mu \mathrm{M})^{[58]}$. Nrf2 can also be activated via Ceramide-PKC $\zeta$-casein kinase 2 signaling pathway that upregulate antioxidant enzymes in the brain ${ }^{[59]}$. The activation of $\mathrm{Nrf} 2$ prevents the transcription of proinflammatory mediators such as TNF- $\alpha$, interleukin-1 (IL-1), inducible nitric oxide synthase (iNOS), interleukin-6 (IL-6), cyclooxygenase-2 (COX-2) and intracellular adhesion molecule $(\mathrm{ICAM})^{[60]}$.

\section{The controversial role of NRF2:}

$\mathrm{Nrf} 2$ is reported to play a crucial role in human carcinogenesis. It has been reported that lung cancer and non-small cell lung cancer (NSCLC) results due to the activation of MEK1/2-ERK1/2-induced apoptosis pathway in endoplasmic reticulum (ER) stress via the activation of unfolded protein response

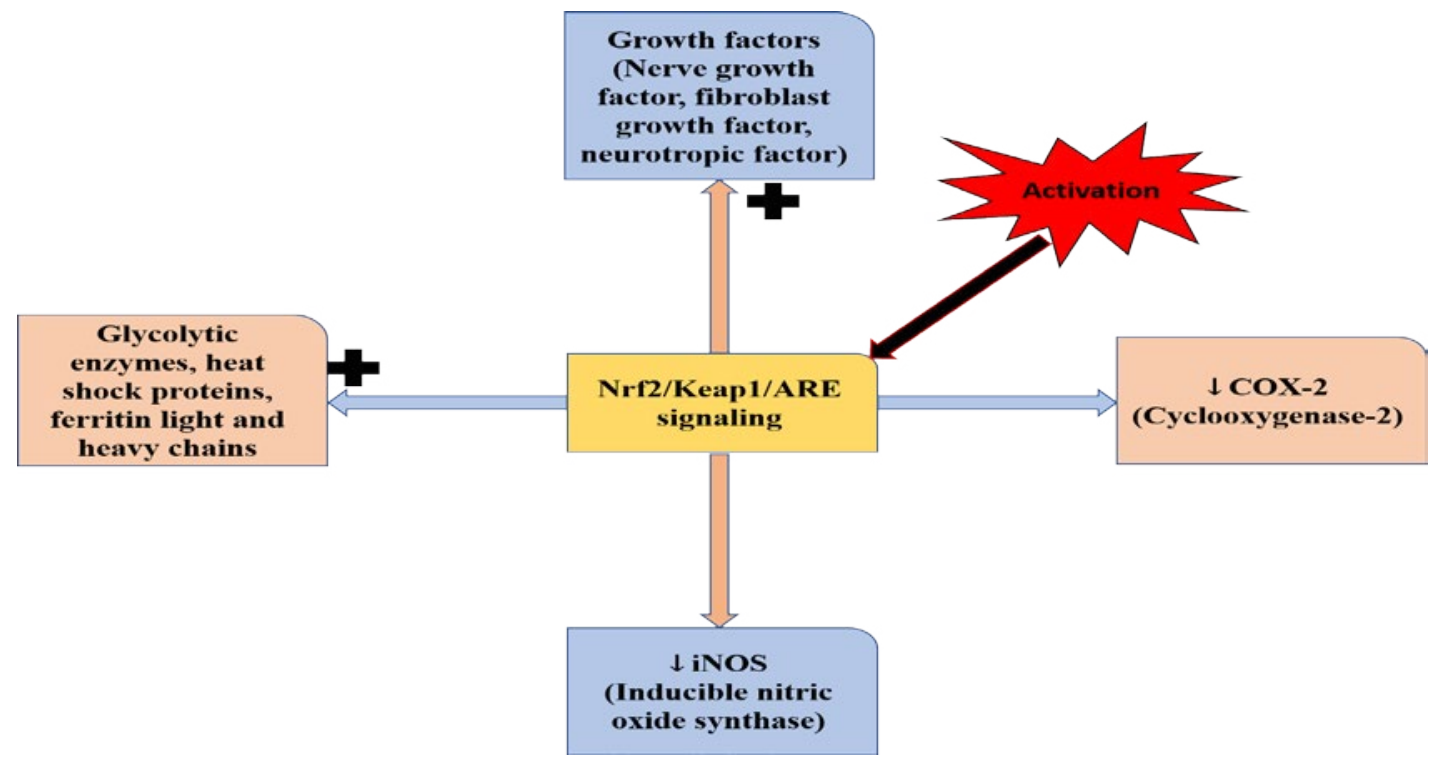

Fig. 7: Activation of Nrf2 by synthetic triterpenoid CDDO-MA 
(UPR). Gall bladder cancer is reported to be caused due to the mutations in Keap1 that leads to the nuclear accumulation of Nrf2 leading to a surge in the expression and activity of genes like NQO1 and GST in the case of Keap1-mutated lung tumors ${ }^{[61,62]}$. In a similar study in NSCLC, by using a combination of erastin and acetaminophen, the regulation of $\mathrm{Nrf2/}$ HO-1 signalling pathways has led to ferroptosis due to the overgeneration of lipid peroxidation ${ }^{[63,64]}$. In a study on head and neck cancer (HNC) using HNC cell lines, it was found out that the overexpression of $\mathrm{Nrf} 2$ leads to chemoresistance of $\mathrm{HN} 3$ cells to Glutathione peroxidase 4 (GPX4)-inhibitor, RSL3 ${ }^{[65]}$. Similarly, increased nuclear expression of $\mathrm{Nrf} 2$ and decreased cytoplasmic expression of Keap1 has been reported in a study conducted among Uighur women associated with cervical squamous cell carcinoma (CSCC) and cervical intraepithelial neoplasia (CIN) due to hypermethylation of $\mathrm{CpG}$ islands in the Keap1 gene promoter and further suggested that in cervical cancer tissues epigenetic changes regulate Keap1 expression ${ }^{[66]}$. These evidences highlights the controversial role of Nrf2 in cancer as it shows protective action in normal and pre-malignant tissues against cancer initiation and progression whereas it enhances the growth of malignant cells ${ }^{[67]}$. Similarly, in myeloid cells of mice where a high expression of Nrf2 occurs specifically in neutrophils, Nrf2 showed a positive effect in chronic colitis whereas, aggravation of the diseases occurs in acute colitis. These leads to a conclusion that the beneficial effects of Nrf2 activation depends on the organ affected, duration of inflammation, stage of the disease and injury model ${ }^{[68-71]}$.

\section{Conclusion and future perspectives:}

ROS are derived mainly from mitochondria. So mitochondrial dysfunctions are often associated with neurodegenerative diseases such as AD, PD, HD and amyotrophic lateral sclerosis. In vivo and in vitro data have identified that phytochemicals can defend cells against OS-induced cellular damage through activation of Nrf2. This suggests that potential therapeutic targeting of Nrf2 could be beneficial for patients associated with neurodegenerative diseases. The role of Nrf2 in cancer is controversial and therefore thorough studies are required to establish the exact role of Nrf2 in pathogenesis of cancer. Nrf2 activators can play a beneficial therapeutic role in prevention and treatment of diseases associated with OS and in cancer and compounds that inhibit Nrf2 could play a role in chemotherapy of cancer.

\section{CONFLICT OF INTEREST}

The authors declare no conflict of interest.

\section{REFERENCES}

1. Suzuki T, Muramatsu A, Saito R, Iso T, Shibata T, Kuwata $\mathrm{K}$, et al. Molecular Mechanism of Cellular Oxidative Stress Sensing by Keap1. Cell Rep 2019;28(3):746-58.

2. Abreu CC, Cardozo LF, Mafra D. Could physical exercises modulate Nrf2-Keap1 pathway in chronic kidney disease? Med Hypotheses. 2015; 84(1):44-6.

3. Adibhatla RM, Hatcher JF. Lipid oxidation and peroxidation in CNS health and disease: from molecular mechanisms to therapeutic opportunities. Antioxid Redox Signal 2010;12(1):125-69.

4. Abudawood M, Tabassum H, Ali MN, Almaarik B, Alshaikh $\mathrm{M}$, et al. Interrelationship between oxidative stress, DNA damage and cancer risk in Diabetes (Type 2) in Riyadh, KSA. Saudi J Biol Sci 2020; 27(1):177-83.

5. Joshi G, A Johnson J. The Nrf2-ARE pathway: a valuable therapeutic target for the treatment of neurodegenerative diseases. Recent Pat CNS Drug Discov 2012;7(3):218-29.

6. Lassmann H, van Horssen J. Oxidative stress and its impact on neurons and glia in multiple sclerosis lesions. Biochim Biophys Acta 2016;1862(3):506-10.

7. Wang Y, Miao X, Sun J, Cai L. Oxidative Stress in Diabetes: Molecular Basis for Diet Supplementation. Mole Nutr Diab 2016; pp.65-72.

8. Ma J, Yang L, Ren J, Yang J. Autophagy, Oxidative Stress, and Redox Regulation. Autophagy Cardiometa Dise 2018; pp: 237-51.

9. Gan L, Johnson JA. Oxidative damage and the Nrf2-ARE pathway in neurodegenerative diseases. Biochim Biophys Acta 2014;1842(8):1208-18.

10. Loboda A, Damulewicz M, Pyza E, Jozkowicz A, Dulak J. Role of Nrf2/HO-1 system in development, oxidative stress response and diseases: an evolutionarily conserved mechanism. Cell Mol Life Sci 2016; 73(17):3221-47.

11. Chapter in a book: Cole MP, Freeman BA. Fatty Acid Transduction of Nitric Oxide Signaling: Cyclooxygenases, Lipoxygenases and Nitro-Fatty Acids. In Nitric Oxide Academic press 2010. p. 391-414.

12. Li X, Wang H, Gao Y, Li L, Tang C, Wen G, et al. Protective effects of quercetin on mitochondrial biogenesis in experimental traumatic brain injury via the Nrf2 signaling pathway. PLoS One 2016;11(10):e0164237.

13. Ma Q. Role of nrf2 in oxidative stress and toxicity. Annual review of pharmacology and toxicology. 2013;53:401-26.

14. Leinonen HM, Kansanen E, Pölönen $P$, Heinäniemi $M$, Levonen AL. Role of the Keap1-Nrf2 pathway in cancer. Adv Cancer Res 2014;122:281-320.

15. Kim KM, Ki SH. Nrf2: a key regulator of redox signaling in liver diseases. In Liver Pathophysiology Academic Press 2017. p. 355-74.

16. Gagné F. Oxidative Stress. Biochemical Ecotoxicology 1st ed. Elsevier; 2014. p. 103-15.

17. Covas G, Marinho HS, Cyrne L, Antunes F. Activation of Nrf2 by H2O2: de novo synthesis versus nuclear translocation. Methods Enzymol 2013;528:157-71.

18. Robledinos-Antón N, Fernández-Ginés R, Manda G, Cuadrado A. Activators and inhibitors of NRF2: a review of their 
potential for clinical development. Oxid Med Cell Longev 2019:9372182.

19. Lehman-McKeeman LD. Biochemical and Molecular Basis of Toxicity. Haschek and Rousseaux's Handbook of Toxicologic Pathology 3rd ed. Elsevier; 2013. p.15-38.

20. David JA, Rifkin WJ, Rabbani PS, Ceradini DJ. The Nrf2/ Keap1/ARE pathway and oxidative stress as a therapeutic target in type II diabetes mellitus. J Diabetes Res 2017;2017: 4826724

21. Itoh K, Wakabayashi N, Katoh Y, Ishii T, Igarashi K, Engel $\mathrm{JD}$, et al. Keap1 represses nuclear activation of antioxidant responsive elements by Nrf2 through binding to the aminoterminal Neh2 domain. Genes Dev 1999;13(1):76-86.

22. Hagemann TL, Jobe EM, Messing A. Genetic ablation of Nrf2/antioxidant response pathway in Alexander disease mice reduces hippocampal gliosis but does not impact survival. PloS one 2012;7(5):e37304.

23. Johnson DA, Johnson JA. Nrf--a therapeutic target for the treatment of neurodegenerative diseases. Free Radic Biol Med 2015;88:253-67.

24. Cuadrado A. NRF2 in neurodegenerative diseases. Current Opinion in Toxicol 2016;1:46-53.

25. Niture SK, Khatri R, Jaiswal AK. Regulation of Nrf2-an update. Free Radic Biol Med 2014;66:36-44.

26. Bhakkiyalakshmi E, Sireesh D, Rajaguru P, Paulmurugan R, Ramkumar KM. The emerging role of redox-sensitive Nrf2Keap1 pathway in diabetes. Pharmacol Res 2015;91:104-14.

27. Zhang W, Liu HT. MAPK signal pathways in the regulation of cell proliferation in mammalian cells. Cell Res 2002;12(1):918.

28. Ferrándiz ML, Nacher-Juan J, Alcaraz MJ. Nrf2 as a therapeutic target for rheumatic diseases. Biochem Pharmacol 2018;152:338-46.

29. Benzie IF, Choi SW. Antioxidants in food: content, measurement, significance, action, cautions, caveats, and research needs. Adv Food Nutr Res 2014;71:1-53.

30. Dasgupta A, Klein K. Oxidative Stress Related to Other Diseases. Antioxida food Vita Supplements 2014; p:185-207.

31. David JA, Rifkin WJ, Rabbani PS, Ceradini DJ. The Nrf2/ Keap1/ARE pathway and oxidative stress as a therapeutic target in type II diabetes mellitus. J Diabetes Res 2017;2017:4826724.

32. Liu B, Shen LJ, Zhao TX, Sun M, Wang JK, Long CL, et al. Automobile exhaust-derived PM2. 5 induces blood-testis barrier damage through ROS-MAPK-Nrf2 pathway in sertoli cells of rats. Ecotoxicol Environ Saf 2020;189:110053.

33. Zhao TX, Wang JK, Shen LJ, Long CL, Liu B, Wei Y, et al. Increased m6A RNA modification is related to the inhibition of the Nrf2-mediated antioxidant response in di-(2-ethylhexyl) phthalate-induced prepubertal testicular injury. Environ Pollut 2020;259:113911.

34. Buendia I, Michalska P, Navarro E, Gameiro I, Egea J, Leon R. Nrf2-ARE pathway: an emerging target against oxidative stress and neuroinflammation in neurodegenerative diseases. Pharmacol Ther 2016;157:84-104.

35. Kerr F, Sofola-Adesakin O, Ivanov DK, Gatliff J, PerezNievas BG, Bertrand HC, et al. Direct Keap1-Nrf2 disruption as a potential therapeutic target for Alzheimer's disease. PLoS Genet 2017;13(3):e1006593.

36. Jin YN, Yanxun VY, Gundemir S, Jo C, Cui M, Tieu K, et al. Impaired mitochondrial dynamics and Nrf2 signaling contribute to compromised responses to oxidative stress in striatal cells expressing full-length mutant huntingtin. PloS one 2013;8(3):e57932.
37. Tocmo R, Parkin K. S-1-propenylmercaptocysteine protects murine hepatocytes against oxidative stress via persulfidation of Keap1 and activation of Nrf2. Free Radic Biol Med 2019;143:164-75.

38. Dhouib IE, Jallouli M, Annabi A, Gharbi N, Elfazaa S, Lasram MM. A minireview on N-acetylcysteine: an old drug with new approaches. Life Sci 2016;151:359-63.

39. Li Y, Paonessa JD, Zhang Y. Mechanism of chemical activation of Nrf2. PloS one 2012;7(4):e35122.

40. Al-Sawaf O, Clarner T, Fragoulis A, Kan YW, Pufe T, Streetz $\mathrm{K}$, et al. Nrf2 in health and disease: current and future clinical implications. Clin Sci (Lond) 2015;129(12):989-99.

41. Chun KS, Kundu J, Kundu JK, Surh YJ. Targeting Nrf2-Keap1 signaling for chemoprevention of skin carcinogenesis with bioactive phytochemicals. Toxic lett 2014;229(1):73-84.

42. Ellrichmann G, Petrasch-Parwez E, Lee DH, Reick C, Arning L, Saft C, et al. Efficacy of fumaric acid esters in the R6/2 and YAC128 models of Huntington's disease. PloS one 2011;6(1):e16172.

43. Ahuja M, Kaidery NA, Yang L, Calingasan N, Smirnova N, Gaisin A, et al. Distinct Nrf2 signaling mechanisms of fumaric acid esters and their role in neuroprotection against 1-methyl4-phenyl-1, 2, 3, 6-tetrahydropyridine-induced experimental Parkinson's-like disease. J Neurosci 2016;36(23):6332-51.

44. Zhou J, Qu XD, Li ZY, Liu Q, Ma YH, He JJ, et al. Salvianolic acid $B$ attenuates toxin-induced neuronal damage via Nrf2-dependent glial cells-mediated protective activity in Parkinson's disease models. PLoS One 2014;9(7):e101668.

45. Ryu J, Zhang R, Hong BH, Yang EJ, Kang KA, Choi M, et al. Phloroglucinol attenuates motor functional deficits in an animal model of Parkinson's disease by enhancing Nrf2 activity. PloS one 2013;8(8):e71178.

46. Zheng Y, Zhu G, He J, Wang G, Li D, Zhang F. Icariin targets Nrf2 signaling to inhibit microglia-mediated neuroinflammation. Int Immunopharmacol 2019;73:304-11.

47. Zou Y, Lee J, Nambiar SM, Hu M, Rui W, Bao Q, et al. Nrf2 is involved in maintaining hepatocyte identity during liver regeneration. PLoS One 2014;9(9):e107423.

48. Zhang YK, Wu KC, Klaassen CD. Genetic activation of Nrf2 protects against fasting-induced oxidative stress in livers of mice. PloS one 2013;8(3):e59122.

49. Hao E, Lang F, Chen Y, Zhang H, Cong X, Shen X, et al. Resveratrol alleviates endotoxin-induced myocardial toxicity via the Nrf2 transcription factor. PloS One 2013;8(7):e69452.

50. Ma B, Zhang J, Zhu Z, Zhao A, Zhou Y, Ying H, et al. Luteolin Ameliorates Testis Injury and Blood-Testis Barrier Disruption through the Nrf2 Signaling Pathway and by Upregulating Cx43. Mol Nutr Food Res 2019;63(10):1800843.

51. Xing $\mathrm{Y}$, Niu $\mathrm{T}$, Wang $\mathrm{W}, \mathrm{Li} \mathrm{J}$, Li S, Janicki JS, et al. Triterpenoid dihydro-CDDO-trifluoroethyl amide protects against maladaptive cardiac remodeling and dysfunction in mice: a critical role of Nrf2. PLoS One 2012;7(9):e44899.

52. Pan H, He M, Liu R, Brecha NC, Yu AC, Pu M. Sulforaphane protects rodent retinas against ischemia-reperfusion injury through the activation of the Nrf2/HO-1 antioxidant pathway. PloS one 2014;9(12):e114186.

53. Chen S, Zhu Y, Liu Z, Gao Z, Li B, Zhang D, et al. Grape seed proanthocyanidin extract ameliorates diabetic bladder dysfunction via the activation of the Nrf2 pathway. PLoS One 2015;10(5):e0126457.

54. Furuya AK, Sharifi HJ, Jellinger RM, Cristofano P, Shi B, de Noronha CM. Sulforaphane inhibits HIV infection of macrophages through Nrf2. PLoS Pathog 2016;12(4):e1005581. 
55. Jazwa A, Rojo AI, Innamorato NG, Hesse M, Fernández-Ruiz J, Cuadrado A. Pharmacological targeting of the transcription factor Nrf2 at the basal ganglia provides disease modifying therapy for experimental parkinsonism. Antioxid Redox Signal 2011;14(12):2347-60.

56. Yang L, Calingasan NY, Thomas B, Chaturvedi RK, Kiaei $\mathrm{M}$, Wille EJ, et al. Neuroprotective effects of the triterpenoid, CDDO methyl amide, a potent inducer of Nrf2-mediated transcription. PLoS One 2009;4(6):e5757.

57. Lin X, Bai D, Wei Z, Zhang Y, Huang Y, Deng H, et al. Curcumin attenuates oxidative stress in RAW264. 7 cells by increasing the activity of antioxidant enzymes and activating the Nrf2-Keap1 pathway. PLoS One 2019;14(5):e0216711.

58. Ishii T, Warabi E, Mann GE. Circadian control of $\mathrm{p} 75$ neurotrophin receptor leads to alternate activation of $\mathrm{Nrf} 2$ and c-Rel to reset energy metabolism in astrocytes via brain-derived neurotrophic factor. Free Radic Biol Med 2018;119:34-44.

59. Bhakkiyalakshmi E, Sireesh D, Ramkumar KM. Redox Sensitive Transcription via Nrf2-Keap1 in Suppression of Inflammation. In: Jungraithmayr W, Bagchi D, editors. Immunity and Inflammation in Health and Disease. 1st ed. Cambridge: Academic Press, 2018. p. 149-61.

60. Joo JH, Jetten AM. Molecular mechanisms involved in farnesol-induced apoptosis. Cancer Lett 2010;287(2):123-35.

61. Aoki Y. Nrf2 as a Possible Determinant of the Threshold for Carcinogenesis. In: Nohmi T, Fukushima S, editors. Thresholds of Genotoxic Carcinogens. 1st ed. Cambridge: Academic Press, 2016. p. 155-70.

62. Gai C, Yu M, Li Z, Wang Y, Ding D, Zheng J, et al. Acetaminophen sensitizing erastin-induced ferroptosis via modulation of $\mathrm{Nrf} 2 /$ heme oxygenase- 1 signaling pathway in non-small-cell lung cancer. J Cell Physiol 2020;235(4):332939.

63. Dodson M, Castro-Portuguez R, Zhang DD. NRF2 plays a critical role in mitigating lipid peroxidation and ferroptosis. Redox Biol 2019;23:101107.

64. Shin D, Kim EH, Lee J, Roh JL. Nrf2 inhibition reverses resistance to GPX4 inhibitor-induced ferroptosis in head and neck cancer. Free Radic Biol Med 2018;129:454-62.

65. Ma JQ, Tuersun H, Jiao SJ, Zheng JH, Hasim A. Functional role of NRF2 in cervical carcinogenesis. PloS one 2015;10(8):e0133876.

66. Kansanen E, Kuosmanen SM, Leinonen H, Levonen AL. The Keap1-Nrf2 pathway: mechanisms of activation and dysregulation in cancer. Redox Biol 2013;1(1):45-9.

67. Joshi N, Werner S. Nrf2 is highly expressed in neutrophils, but myeloid cell-derived $\mathrm{Nrf} 2$ is dispensable for wound healing in mice. PLoS One 2017;12(10):e0187162.

68. Mesika R, Reichmann D. When safeguarding goes wrong: Impact of oxidative stress on protein homeostasis in health and neurodegenerative disorders. Adv Protein Chem Struct Biol 2019;114(1):221-64.

69. Federico A, Cardaioli E, Da Pozzo P, Formichi P, Gallus GN, Radi E. Mitochondria, oxidative stress and neurodegeneration. J Neurol Sci 2012;322(1-2):254-62.

70. Senger DR, Li D, Jaminet SC, Cao S. Activation of the Nrf2 cell defense pathway by ancient foods: disease prevention by important molecules and microbes lost from the modern western diet. PLoS One 2016;11(2):e0148042.

71. Sporn MB, Liby KT. NRF2 and cancer: the good, the bad and the importance of context. Nat Rev Cancer 2012;12(8):564. 